\title{
NOTE ON RESTRICTION OF FOURIER TRANSFORMS
}

\author{
MEN-CHANG HU $^{1}$ \\ ABSTRaCT. A technique for obtaining necessary conditions on restriction of Fourier
} transforms is introduced.

In [1]. E. Prestini has proved that if $\alpha$ is a compact $C^{3}$ curve in $\mathbf{R}^{3}$ with nonvanishing curvature and torsion, then the inequality

$$
\left\|\left.\hat{f}\right|_{\alpha}\right\|_{L^{4}(\alpha)} \leqslant C_{p}\|f\|_{L^{p}(\mathbf{R})^{3}}, \quad f \in \widehat{S} .
$$

holds if $1 \leqslant p<15 / 13$ and $1 / q>6(1-1 / p)$. The inequality does not hold if $p \geqslant 6 / 5$ or $1 / q<6(1-1 / p)$. In this note we shall elaborate an idea of Knapp to prove that the inequality does not hold if $p>7 / 6$. Our argument, which can be applied in similar situations also, is presented in the following paragraph.

We assume $\alpha$ is defined by the equation $(t, \phi(t), \psi(t)), 0 \leqslant t \leqslant \eta$, where $\eta$ is a small positive number and $\phi(t)=t^{2}+\xi(t), \psi(t)=t^{3}+\zeta(t), \xi(t)$ and $\zeta(t)$ are infinitesimals of third and fourth order w.r.t. $t$. Choose a large positive number $M$. For each positive integer $k$, set $\eta_{k}=2^{-k} \eta$ and $\delta_{k}=\eta_{k} / M$. For each $j=1,2 \ldots, 2^{k}$

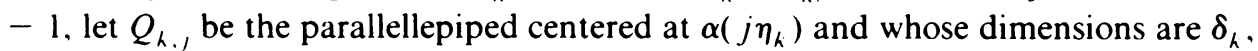
$\delta_{k}^{2}, \delta_{k}^{3}$ along the tangent, normal and binormal at $\alpha\left(j \eta_{k}\right)$, respectively. Note that for $M$ sufficiently large, we may asume that, for each $k$, the collection $\left\{(1+1 / M) Q_{k, j}: 0<j<2^{k}\right\}$ are pairwise disjoint and there exists $\theta>0$ such that, for each $k$ and $j,\left\{\alpha(t):\left|t-j \eta_{k}\right|<\theta \delta_{k}\right\} \subset Q_{k, j}$. Choose a smooth function $g$ such that $g(x)=1$ if $x$ lies in $Q$, the unit cube centered at the origin, and $g(x)=0$ if $x$ lies outside $(1+1 / M) Q$. Put $g_{k}\left(x_{1}, x_{2}, x_{3}\right)=g\left(x_{1} / \delta_{k}, x_{2} / \delta_{k}^{2}, x_{3} / \delta_{k}^{3}\right)$. Performing a suitable rigid motion to $g_{k}$, we obtain a function $g_{k, j}$ such that $g_{k, j}(x)=1$ if $x$ lies in $Q_{k, j}$ and $g_{k, j}(x)=0$ if $x$ lies outside $(1+1 / M) Q_{k, j}$. Let $\hat{f}_{k, j}=g_{k, j}$. Then $\left\|f_{h, j}\right\|_{L^{p}\left(\mathbf{R}^{3}\right)}=\delta_{k}^{6(1-1 / p)}\|\hat{g}\|_{I^{r}\left(\mathbf{R}^{3}\right)}$. Since the functions $f_{k, j}, 0<j<2^{k}$. are rapidly decreasing, there exists points $w_{k, j}, 0<j<2^{k}$, such that

$$
\left\|\sum_{j} \tau_{\mu_{k, j}} f_{k, j}\right\|_{L^{p}\left(\mathbf{R}^{3}\right)}^{p} \leqslant 2 \sum_{j}\left\|\tau_{\mu_{k, j}, f_{k, j}}\right\|_{L^{p}\left(\mathbf{R}^{3}\right)}^{p} .
$$

Note that, for $\left|t-j \eta_{k}\right|<\theta \delta_{k}$ and $0<j<2^{k}$,

$$
\left|\widehat{\sum_{j} \tau_{w_{k, j}, j} f_{k, j}}(\alpha(t))\right|=1 .
$$

Received by the editors June 3, 1982.

1980 Mathematics Subject Classification. Primary 42A68.

'This research was supported in part by the National Science Council, Republic of China. 
If the inequality ( 1 ) is true, we would have

$$
\sum_{j} \int_{j \eta_{k}-\theta \delta_{k}}^{j \eta_{k}+\theta \delta_{k}} 1 \leqslant C\left(\sum_{j} \delta_{k}^{6(p-1)}\right)^{q / p} .
$$

Hence $1 \leqslant C \cdot 2^{k} \cdot 2^{-6(p-1) k}$, for all positive integers $k$. Clearly this can hold only if $p \leqslant 7 / 6$.

REMARK. The above idea is useful even if the curvature vanishes somewhere (see [2]).

\section{REFERENCES}

1. E. Prestini, A restriction theorem for space curves, Proc. Amer. Math. Soc. 70 (1978), 8-10.

2. M. C. Hu, Restriction of Fourier transforms to plane curves (preprint).

institute of Mathematics, Academia Sinica, Taipei, Taiwan, Republic of China 